\title{
Calculation of Beta Decay Half-Lives and Delayed Neutron Branching Ratio of Fission Fragments with Skyrme-QRPA
}

\author{
Futoshi Minato $^{1 \mathrm{a}}$ \\ ${ }^{1}$ Nuclear Data Center, Japan Atomic Energy Agency, Tokai 319-1195, Japan
}

\begin{abstract}
Nuclear $\beta$-decay and delayed neutron (DN) emission is important for the $r$ process nucleosynthesis after the freeze-out, and stable and safe operation of nuclear reactors. Even though radioactive beam facilities have enabled us to measure $\beta$-decay and branching ratio of neutron-rich nuclei apart from the stability line in the nuclear chart, there are still a lot of nuclei which one cannot investigate experimentally. In particular, information on DN is rather scarce than that of $T_{1 / 2}$. To predict $T_{1 / 2}$ and the branching ratios of DN for next JENDL decay data, we have developed a method which comprises the quasiparticle-random-phase-approximation (QRPA) and the Hauser-Feshbach statistical model (HFSM). In this work, we calculate fission fragments with $T_{1 / 2} \leq 50 \mathrm{sec}$. We obtain the rms deviation from experimental half-life of 3.71. Although the result is still worse than GT2 which has been adopted in JENDL decay data, DN spectra are newly calculated. We also discuss further subjects to be done in future for improving the present approach and making next generation of JENDL decay data.
\end{abstract}

\section{Introduction}

Nuclear data center (NDC) at Japan Atomic Energy Agency (JAEA) provides various kinds of nuclear data ${ }^{1}$. One of them is the decay data, which includes information concerning nuclear decay properties such as half-life, branching ratio of delayed neutron (DN), $\beta$-spectra, and so on. The data is practically used to evaluate the amount of radioactivity of unstable nuclei followed by a nuclear reaction and a fission. Such a work is important in the decomissioning of nuclear reactor and astrophysical applications. NDC has released the JENDL fission product decay data in 2011 (JENDL/FPD-2011) [1], which covers decay data of 1,284 nuclides. It is able to reproduce experimentally measured decay heat generated by fission products successfully by incorporating the total absorption gamma spectroscopy (TAGS) data. Recently, NDC started to distribute a new decay data, JENDL decay data file 2015 (JENDL/DDF-2015) [2], from November 2015. The file contains 3,237 nuclides ranging from the lightest elements including $\mathrm{H}-1$ and $\mathrm{n}$, up to $\mathrm{Rf}(\mathrm{Z}=104)$. The decay data is also provided in JEFF [3] and ENDF [4] nuclear data groups. Recent development of accelerators which utilize radioactive beams makes it possible to investigate unstable nuclei which one hasn't been able to study, and the renewal of the decay data becomes also active.

Although the JENDL/FPD-2011 has succeeded to reproduce the decay heats, it is pointed out that it cannot reproduce the total amount of $\mathrm{DN}$ after a nuclear fission. A part of the problem might be

\footnotetext{
a e-mail: minato.futoshi@jaea.go.jp

1 available from our website, http://wwwndc.jaea.go.jp/index.html
} 
attributed to incorrect evaluation of the fission yield data as discussed in Ref. [5], and the other might be due to the accuracy of decay data. Besides the problem, JENDL/FPD-2011 didn't contain the DN spectra, which are considered as one of the promising quantities applicable for nuclear nondestructive inspection (the situation remains unchanged in JENDL/DDF-2015). Experimental data of DN spectra is limited strongly. Although new experiments to investigate DN are planned at several experimental facilities in the world, we still need to depend on theoretical methods to derive most of DN information. In case of JENDL/FPD-2011 and JENDL/DDF-2015, the decay data concerning unmeasured $\beta$-decay are calculated with Gross Theory 2 (GT2) [6], which has been also widely used to discuss and compare with experimental data. However, the approach uses a statistical treatment in the level density so that it cannot provide a detailed nuclear structure (i.e., spin, parity and energy of excited levels), although it is able to reproduce half-lives systematically well. Besides, recent measurements of neutron-rich nucleus indicate that the GT2 fails to reproduce half-lives of some nuclei close to the neutron drip-line [7]. One of the reasons might be due to that GT2 is based on a phenomenological method whose parameters are adjusted by known experimental data. Therefore, it is required to make an alternative theoretical method to calculate data on $\beta$-decay for next JENDL decay data.

To calculate $\beta$-decay and DN, we have developed a new approach, which comprises quasiparticlerandom-phase-approximation (QRPA) and the Hauser-Feshbach statistical model (HFSM) [8]. This approach is capable to calculate $\beta$-decay and DN emission of nuclei on the nuclear chart systematically as well as GT2. In addition, the formalism starts from the two-body effective interaction, and can take into account the nuclear deformation and the pairing effects consistently, so that it can provide a more reliable prediction of half-life for nuclei which have not been measured experimentally. Moreover, it can give a detailed nuclear structure of excited states. The systematical calculations of half-lives and branching ratio of DN using QRPA have been performed not only by non-relativistic but also relativistic approaches [9-11]. Finite amplitude method, which is equivalent to QRPA, was also applied to systematical $\beta$-decay calculation [12]. However, they don't discuss DN spectra. The data of DN spectra is only available in ENDF/B-VII.1 decay data [4], in which FRDM+QRPA is used to calculate unmeasured half-lives [13] and $\mathrm{CoH}$ code to calculate DN spectra [14]. However, our approach is more sophisticated as compared to FRDM+QRPA with respect to full microscopic and respecting self-consistency of the effective interaction in the ground and excited states.

So far, we have examined the reproduction power of our approach. However, we have found that it gave large deviations from experimental half-lives, especially in odd-A and odd-odd nuclei. The reason is clear; we have assumed that valence nucleon(s) is(are) unperturbatively put on the last orbit outside even-even core nuclei and its $\beta$-transition is treated in a manner of the single particle one. In this work, we assess two approaches for better description of odd nuclei, that is, the blocking effect approximation (BEA) and the equal filling approximation (EFA), and re-examined the reproduction power.

\section{Theoretical Method}

To calculate $\beta$-decays and branching ratios of $\mathrm{DN}$, three processes are carried out. At first, the ground state of nucleus is derived with the Skyrme-Hartree-Fock + BCS method. The effective two-body interaction that we used is the SkO' parameter set [15]. The pairing force which accounts for the short-range correlation between identical particles (isospin $T=1$ ) is given in the volume-type form,

$$
v\left(\vec{r}, \overrightarrow{r^{\prime}}\right)=P(S=0) P(T=1) v_{01} \delta\left(\vec{r}-\overrightarrow{r^{\prime}}\right),
$$

where $P(S=0)$ and $P(T=1)$ are the projection operator for $S=0$ and $T=1$, respectively, and $v_{01}$ the parameter representing the pairing strength. Because we adopt the zero-range force as the pairing 
force, a pairing active space is introduced. We use the same approach as the reference [16]. For proton and neutron, we set $v_{01}=256.1,258.1 \mathrm{MeV} \mathrm{fm}^{3}$ [15], respectively. The calculation is performed in the cylindrical-coordinate system, where the axial symmetry is assumed. The single-particle levels in the continuum are taken into account by discretizing them in the boundary box. We use $16 \mathrm{fm}$ with the step size $\Delta z=\Delta \rho=0.8 \mathrm{fm}$ for the boundary condition.

In the second step, excited states of the daughter nucleus are calculated by QRPA based on the ground state calculated by the Skyrme-Hartree-Fock + BCS. The phonon creation operator in our formalism is given by

$$
Q_{v}^{\dagger}=\sum_{p n} X_{p n}^{v} \alpha_{p}^{\dagger} \alpha_{n}^{\dagger}-Y_{p n}^{v} \alpha_{n} \alpha_{p}
$$

and the excited state can be approximated by

$$
|v\rangle=Q_{v}^{\dagger}|\mathrm{QRPA}\rangle \sim \mathrm{Q}_{\nu}^{\dagger}|0\rangle,
$$

where $|v\rangle$ is $v$-th excited state, |QRPA $\rangle$ the QRPA ground state, and $|0\rangle$ the Skyrme-Hartree-Fock + BCS ground state. The coefficients $X$ and $Y$ are derived by solving the QRPA-equation,

$$
\left(\begin{array}{cc}
A & B \\
-B^{*} & -A^{*}
\end{array}\right)\left(\begin{array}{c}
X \\
Y
\end{array}\right)=E_{Q R P A}\left(\begin{array}{c}
X \\
Y
\end{array}\right)
$$

where submatrices $A$ and $B$ are expressed by

$$
\begin{aligned}
A_{p n p^{\prime} n^{\prime}}= & \left(E_{p}+E_{n}\right) \delta_{p p^{\prime}} \delta_{n n^{\prime}}+\left(u_{p} v_{n} u_{p^{\prime}} v_{n^{\prime}}+v_{p} u_{n} v_{p^{\prime}} u_{n^{\prime}}\right)\left\langle p \bar{n}^{\prime}|v| \bar{n} p^{\prime}\right\rangle \\
& +\left(u_{p} u_{n} u_{p^{\prime}} u_{n^{\prime}}+v_{p} v_{n} v_{p^{\prime}} v_{n^{\prime}}\right)\left\langle p n|v| p^{\prime} n^{\prime}\right\rangle \\
B_{p n p^{\prime} n^{\prime}}= & -\left(u_{p} v_{n} v_{p^{\prime}} u_{n^{\prime}}+v_{p} u_{n} u_{p^{\prime}} v_{n^{\prime}}\right)\left\langle p n^{\prime}|v| \bar{n} \bar{p}^{\prime}\right\rangle-\left(u_{p} u_{n} v_{p^{\prime}} v_{n^{\prime}}+v_{p} v_{n} u_{p^{\prime}} u_{n^{\prime}}\right)\left\langle p n|v| \bar{p}^{\prime} \bar{n}^{\prime}\right\rangle
\end{aligned}
$$

The factors, $u_{i}$ and $v_{i}$, are the BCS coefficients. The matrix elements of the particle-hole residual interaction in Eq. (5) are derived self-consistently from the second derivative of energy with respect to the density matrix, while those of the particle-particle one are calculated using the similar force as Eq. (1), given by

$$
v\left(\vec{r}, \vec{r}^{\prime}\right)=P(S=1) P(T=0) v_{10} \delta\left(\vec{r}-\vec{r}^{\prime}\right) .
$$

For the excited states, we consider only the Gamow-Teller transition, and the 1st forbidden transitions are not taken into account in this work, so that $T=1$ pairing force does not take part in the particleparticle channel in Eq. (5). We don't consider $T=0$ pairing when solving the BCS gap equation because its effect on the ground state is considered to be small for $\mathrm{N}>\mathrm{Z}$ nuclei. Therefore, $v_{10}$ in Eq. (6) is treated as a free parameter in our formalism. We determine it by reproducing experimental half-lives of even-even nuclei. Then, we take the average values for each isotopes and use it for systematical calculation. In case of odd-Z nucleus with proton number $Z, v_{10}$ of $Z+1$ nucleus is used assuming that property of even-even core nucleus is not changed much. The parameters $v_{10}$ used in our work are listed in Tab. 1. We introduce the model space of single particle energy up to $20 \mathrm{MeV}$ and two quasi-particle energy up to $60 \mathrm{MeV}$ in solving the QRPA equation.

Once we obtain the excited states of the daughter nucleus, we can calculate the half-life, $T_{1 / 2}$, which is given by,

$$
\frac{1}{T_{1 / 2}}=\frac{\lambda_{\beta}}{\ln 2}=\frac{\left(g_{A} / g_{V}\right)^{2}}{D} \int_{0}^{Q_{\beta}} \sum_{v}\left|\left\langle v\left|\sigma \tau^{-}\right| 0\right\rangle\right|^{2} E_{e} \sqrt{E_{e}^{2}-m_{e}^{2}}\left(E-E_{e}\right)^{2} F\left(Z, E_{e}\right) d E_{e},
$$

where $F\left(Z, E_{e}\right)$ is the Fermi function accounting for the final state interaction between nucleus and outgoing electron and $D=6163.4$ s. The $Q_{\beta}$ values are derived in the same way as Ref. [11]. 
Table 1. Isocaler $T=0$ pairing strength, $v_{10}$ (in unit of $\mathrm{MeV} \mathrm{fm}^{3}$ ), used in this work.

\begin{tabular}{c|ccccccccc}
\hline Element & $\mathrm{Fe}$ & $\mathrm{Co}$ & $\mathrm{Ni}$ & $\mathrm{Cu}$ & $\mathrm{Zn}$ & $\mathrm{Ga}$ & $\mathrm{Ge}$ & $\mathrm{As}$ & $\mathrm{Se}$ \\
& 0 & 380 & 380 & 208 & 208 & 175 & 175 & 0 & 0 \\
\hline Element & $\mathrm{Br}$ & $\mathrm{Kr}$ & $\mathrm{Rb}$ & $\mathrm{Sr}$ & $\mathrm{Y}$ & $\mathrm{Zr}$ & $\mathrm{Nb}$ & $\mathrm{Mo}$ & $\mathrm{Tc}$ \\
& 68 & 68 & 0 & 0 & 0 & 0 & 0 & 0 & 0 \\
\hline Element & $\mathrm{Ru}$ & $\mathrm{Rh}$ & $\mathrm{Pd}$ & $\mathrm{Ag}$ & $\mathrm{Cd}$ & $\mathrm{In}$ & $\mathrm{Sn}$ & $\mathrm{Sb}$ & $\mathrm{Te}$ \\
& 0 & 160 & 160 & 303 & 303 & 220 & 220 & 75 & 75 \\
\hline Element & $\mathrm{I}$ & $\mathrm{Xe}$ & $\mathrm{Cs}$ & $\mathrm{Ba}$ & $\mathrm{La}$ & $\mathrm{Ce}$ & $\mathrm{Pr}$ & $\mathrm{Nd}$ & $\mathrm{Pm}$ \\
& 0 & 0 & 0 & 0 & 190 & 190 & 0 & 0 & 260 \\
\hline Element & $\mathrm{Sm}$ & $\mathrm{Eu}$ & $\mathrm{Gd}$ & $\mathrm{Tb}$ & $\mathrm{Dy}$ & & & & \\
& 260 & 380 & 380 & 0 & 0 & & & & \\
\hline
\end{tabular}

In the third step, the neutron emission from the excited states are calculated. We assume that the daughter nucleus is a compound nucleus and apply the Hausher-Feshbach statistical model, which is implemented in the code CCONE [17], to estimate neutron evaporation probabilities. The global neutron optical potential of Ref. [18] and EGLO model are used to calculate neutron and $\gamma$ transmission coefficients, respectively. For level densities, the formula of Gilbert and Cameron [19] is used, and the parameters are taken from Mengoni-Nakajima's [20]. Then, we obtain the neutron emission probability, $P_{n}^{v}$, and $\gamma$ emission probability, $P_{\gamma}^{v}$, from the $v$-th excited state. The branching ratios of delayed neutron are then calculated by the relation

$$
P_{\beta n}=\frac{\sum_{v} \lambda_{v} P_{n}^{v}}{\sum_{v} \lambda_{v}}
$$

where $\lambda_{v}=\ln 2 / T_{1 / 2, v}$ are the $\beta$-decay rate from the ground state of parent nucleus to $v$-th excited state of daughter nucleus. CCONE code also provide a neutron spectrum from an excited state so that we can obtain a DN spectrum by summing them over the excited states. The spectra is normalized as done in Ref. [14].

\subsection{Odd-A and Odd-Odd nuclei}

To calculate odd-A and odd-odd nuclei, we tested the blocking effect approximation (BEA) [21] and the equal filling approximation (EFA) [22], for the ground state. For BEA, we assume the time reversal symmetry, namely the state occupied by a valence particle, which corresponds to the blocking state, have $u_{i}^{2}=u_{\vec{i}}^{2}=v_{i}^{2}=v_{\vec{i}}^{2}=0.5$. The ground state is sought by blocking single particle levels near the Fermi energy one by one to find the lowest total energy. For EFA, the obtained BCS coefficients, which satisfy the odd particle number, are also used in QRPA. Both for BEA and EFA, we omit the coupling between valence particle and core nucleus in calculating QRPA.

\section{Result}

We first discuss the result of half-lives calculated by EFA and BEA. Figure 1 shows the half-lives of nickel and cobalt isotopes, comparing with the experimental data taken from [23]. For nickel isotopes, the calculations both for EFA and BEA reproduce the experimental data reasonably. For cobalt isotopes, EFA provides the half-lives in the same order as the experimental data, while BEA underestimates them strongly. The half-life is not only governed by $\beta$-transition of core nucleus 

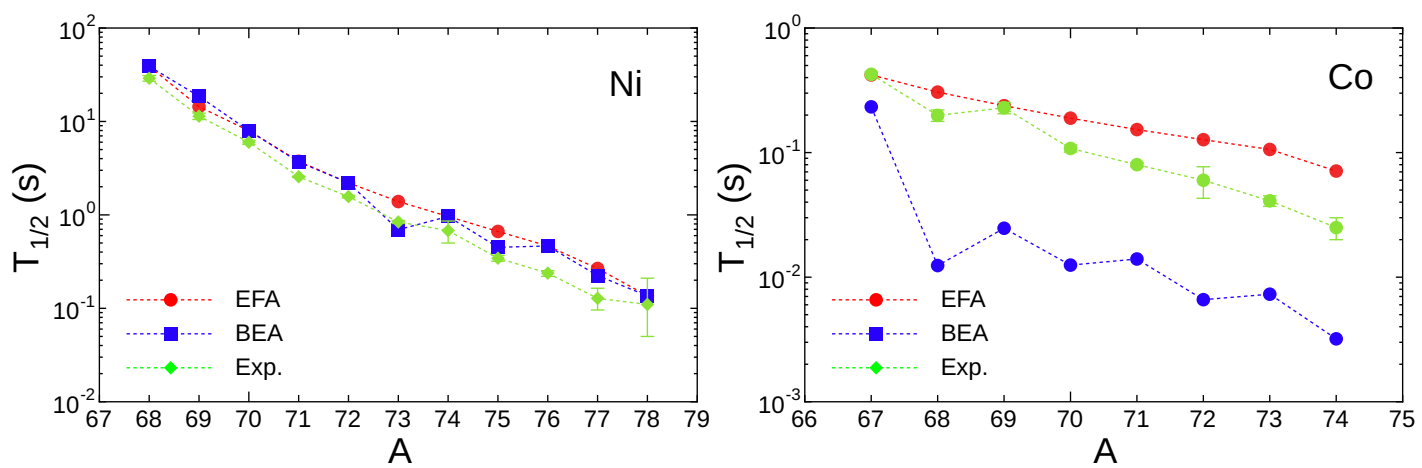

Figure 1. Half-lives of nickel and cobalt isotopes calculated by EFA and BEA, comparing with the experimental data.

but also that of valence nucleon. As a consequence, the half-life changes significantly depending on which orbit the valence nucleon occupies for BEA. On the other hand, since EFA averages the occupation probability of valence nucleon over the orbits near the Fermi energy, contribution of the valence nucleon to the half-life is less sensitive than BEA. In the present approach, the ground state is sought for the lowest total energy, but we omit higher-order effects caused by rotational and vibrational coupling. If we would include those effects, we would obtain a better result. However, it requires a more sophisticated formulation and a much longer computational time. For further improvement of the decay data evaluation, it will be important. In the following, $\beta$-decay and DN are calculated with EFA approach, which gives a more consistent result to experimental data than BEA.

Figures 2 and 3 show the results of half-lives of $Z=26 \sim 33$ and $Z=34 \sim 41$, respectively, calculated by the present approach. For comparison, KTUY+GT2 [6] (hereafter, GT2) and experimental data taken from [23] are also shown. From $Z=26$ to 33 shown in Fig. 2, the present approach is comparable to GT2. On the other hand, the present approach shows shorter half-lives than GT2 systematically from $Z=34$ to 41 shown in Fig. 3 and GT2 is relatively closer to experimental data than the present approach except $\mathrm{Rb}$ isotopes. We notice that from neutron number $N=64$, the present approach shows dumps, which give deviations from experimental data as well as GT2. At the moment, we don't find the physical answer making this dump. This point is the subject to be remedied in future.

Figures 4 and 5 show the results of branching ratio of DN for $Z=26 \sim 33$ and $Z=34 \sim$ 41 , respectively, comparing with GT2. The result of the present work shows a sudden increase and decrease, while GT2 shows a monotonic increase as a function of the mass number $A$ and roughly follows the experimental data. The defect of the present work in the branching ratio is, of course, due to incorrect reproduction of $\beta$-strength function. However, this disappointing result might be improved by including the 1st forbidden transition. As discussed in the paper [9], the 1st forbidden transition produces an additional $\beta$-strength in low energy region below neutron threshold, and then the $\mathrm{DN}$ emission probabilities are reduced to a moderate value. 

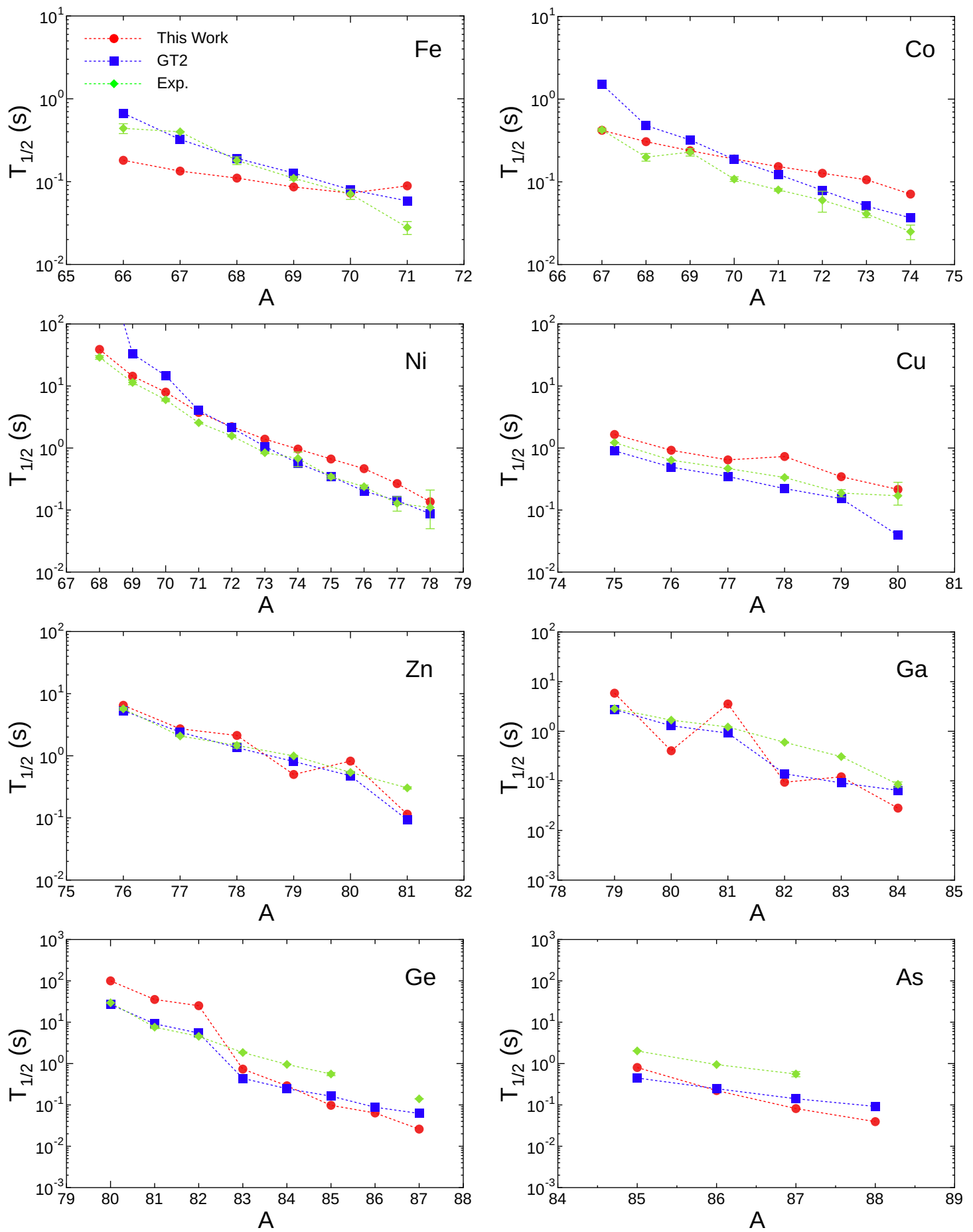

Figure 2. Half-lives of $Z=26$ to 33 nuclei. The result of BEA and EFA are shown. 

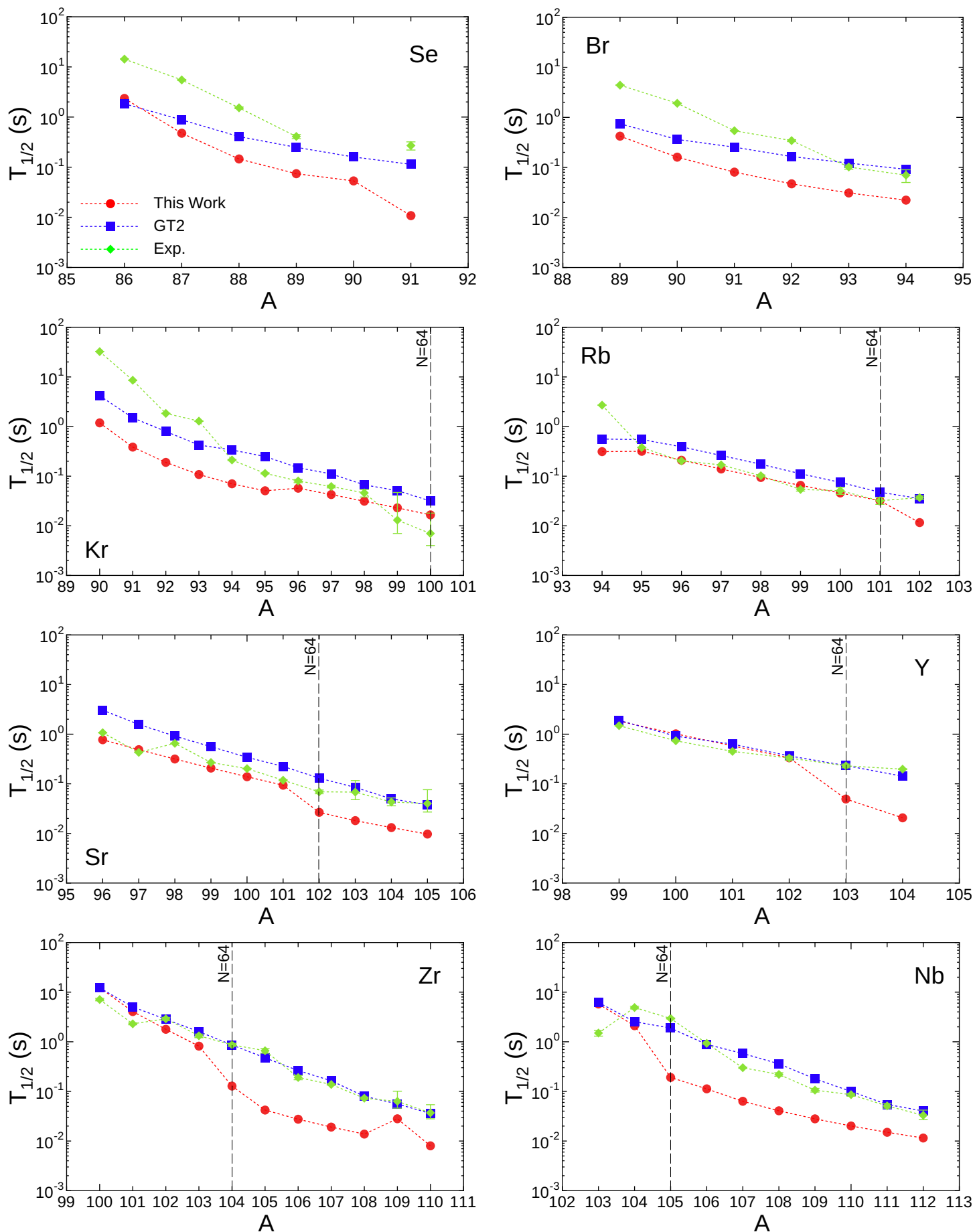

Figure 3. Same as Fig. 2, but for $Z=34$ to 41 nuclei. The vertical line in the panels indicates $N=64$. 

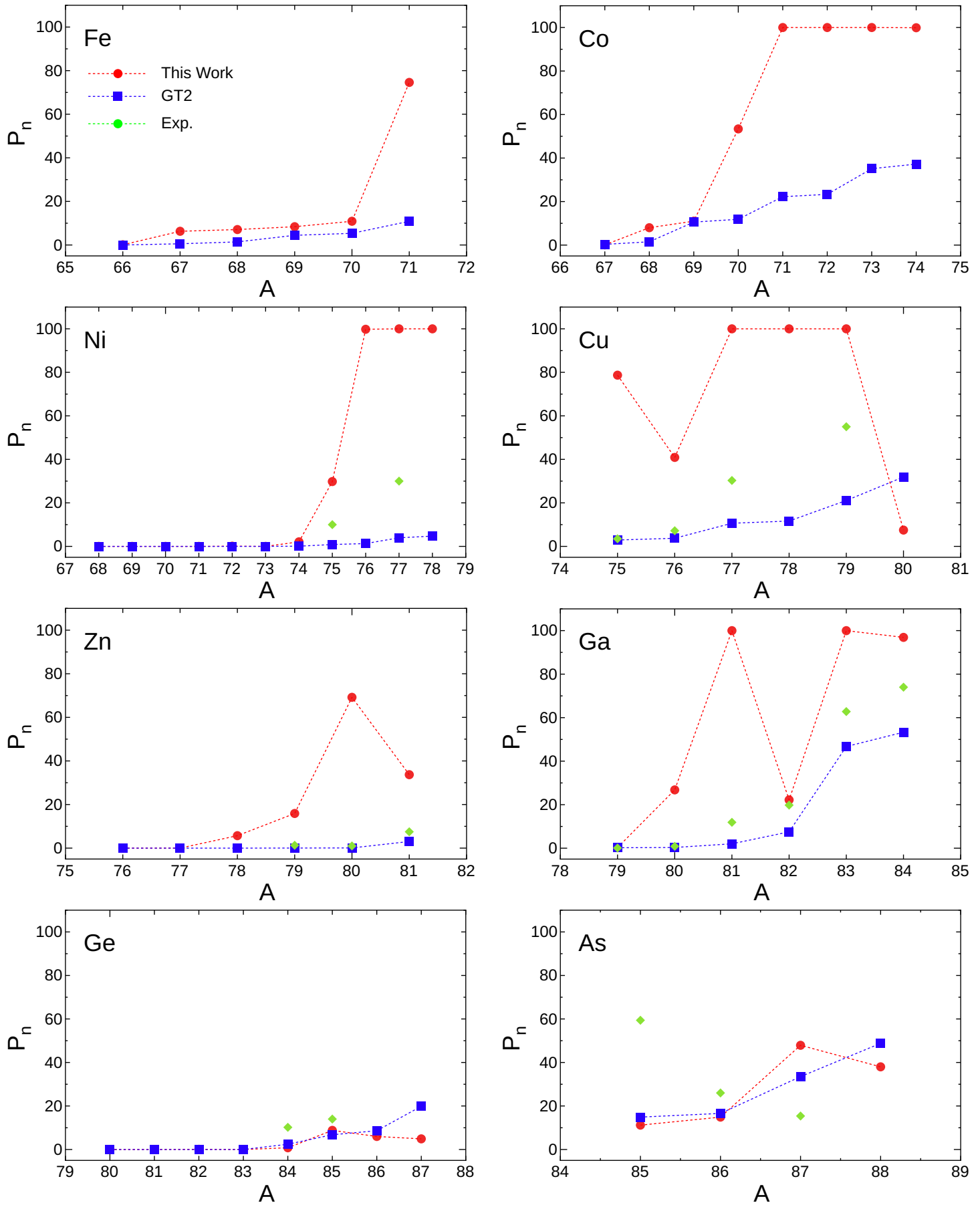

Figure 4. Calculated branching ratios of $\mathrm{DN}$ for $Z=26$ to 33 nuclei, comparing with the experimental data taken from [23] and GT2. 

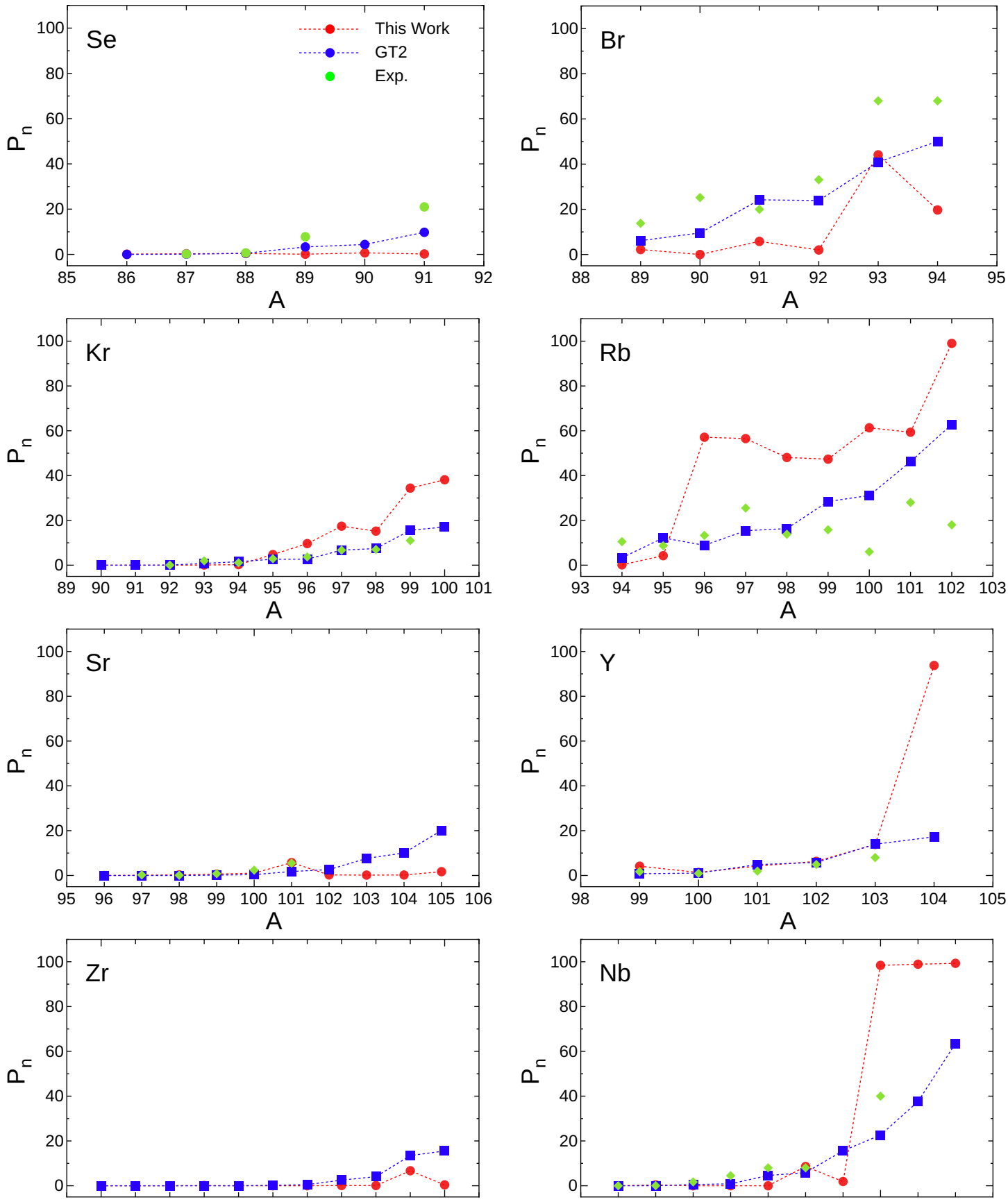

A

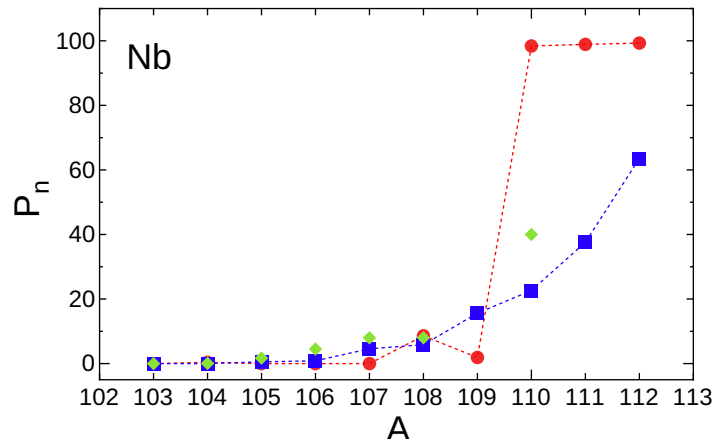

Figure 5. Same as fig. 5, but for $Z=34$ to 41 nuclei. 

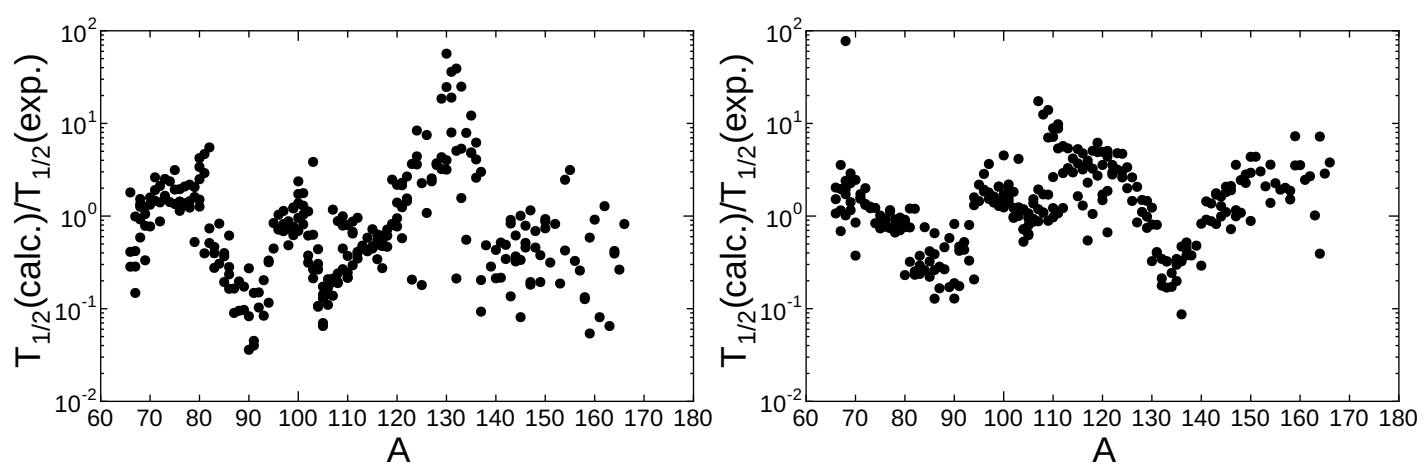

Figure 6. Ratio of the half-lives (the top panels) and the branching ratios (the bottom panels) to the experimental data. The left panels are for the present result, and the right ones are for the GT2.

Figure 6 shows the ratio of the half-lives and the branching ratios defined by

$$
\begin{aligned}
& \sigma_{r m s}\left(T_{1 / 2}\right)=\exp \left(\sqrt{\frac{1}{N} \sum_{i}^{N} \log \left(\frac{T_{1 / 2}(t h .)}{T_{1 / 2}(\exp .)}\right)}\right) \\
& \sigma_{r m s}\left(P_{\beta n}\right)=\exp \left(\sqrt{\frac{1}{N} \sum_{i}^{N} \log \left(\frac{P_{\beta n}(t h .)}{P_{\beta n}(\exp .)}\right)}\right) .
\end{aligned}
$$

For 280 nuclei from $Z=24$ to 64 with $T_{1 / 2} \leq 50 \mathrm{sec}$, the present work provides $\sigma_{R M S}\left(T_{1 / 2}\right)=$ 3.71, which is improved well as compared to 4.90 of the previous work [8]. On the other hand, for 114 nuclei whose experimental data are available, $\sigma_{R M S}\left(P_{\beta n}\right)=9.44$ is not improved so much (in the previous work, 9.85). GT2 gives a better value, $\sigma_{R M S}\left(T_{1 / 2}\right)=2.76$ and $\sigma_{R M S}\left(P_{\beta n}\right)=3.16$. At the moment, the present approach doesn't take into account the 1st forbidden transition, on the other hand, GT2 does. The better quality of GT2 might be due to this point. Therefore, we plan to include the 1 st forbidden transition now.

Finally, Figs. 7 and 8 illustrate the DN spectra of free chosen nuclei, in which the results are compared with those given in the ENDF/B-VII.1 decay data library. Despite the theoretical approaches calculating $\beta$-strength function are different, the spectra of ENDF and our approach are similar for most of calculated nuclei. As we mentioned above, if we take into account the 1 st forbidden transition, the result might be changed.

\section{Conclusion}

We have developed the method which combines QRPA and HFSM. To improve the description of odd-A and odd-odd nuclei, we examined the blocking effect approximation and the equal filling approximation in the ground state. We found that the latter method gives a better reproduction in halflives systematically. However, the reproduction power of the present approach both for half-lives and branching ratios of delayed neutron was still worse than that of GT2. This deviation may be attributed from the omission of 1st forbidden transition in our formalism, which becomes more effective as the neutron and proton shell gaps become larger for neutron-rich side as well as heavier nuclei. We also omitted the coupling between valence particle(s) and core nucleus, i.e., core polarization effect. We 

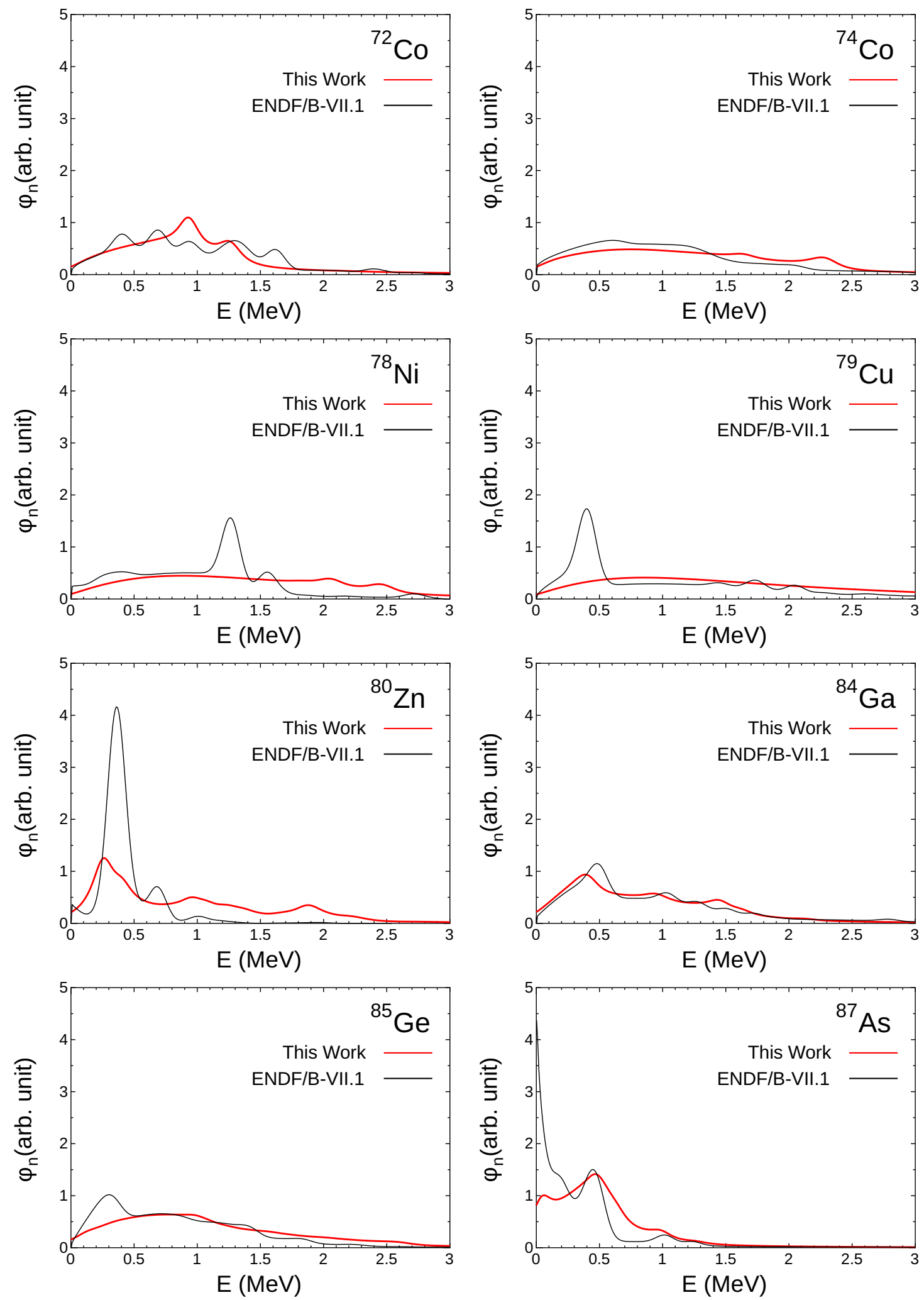

Figure 7. Delayed neutron spectra for free chosen nuclei. 

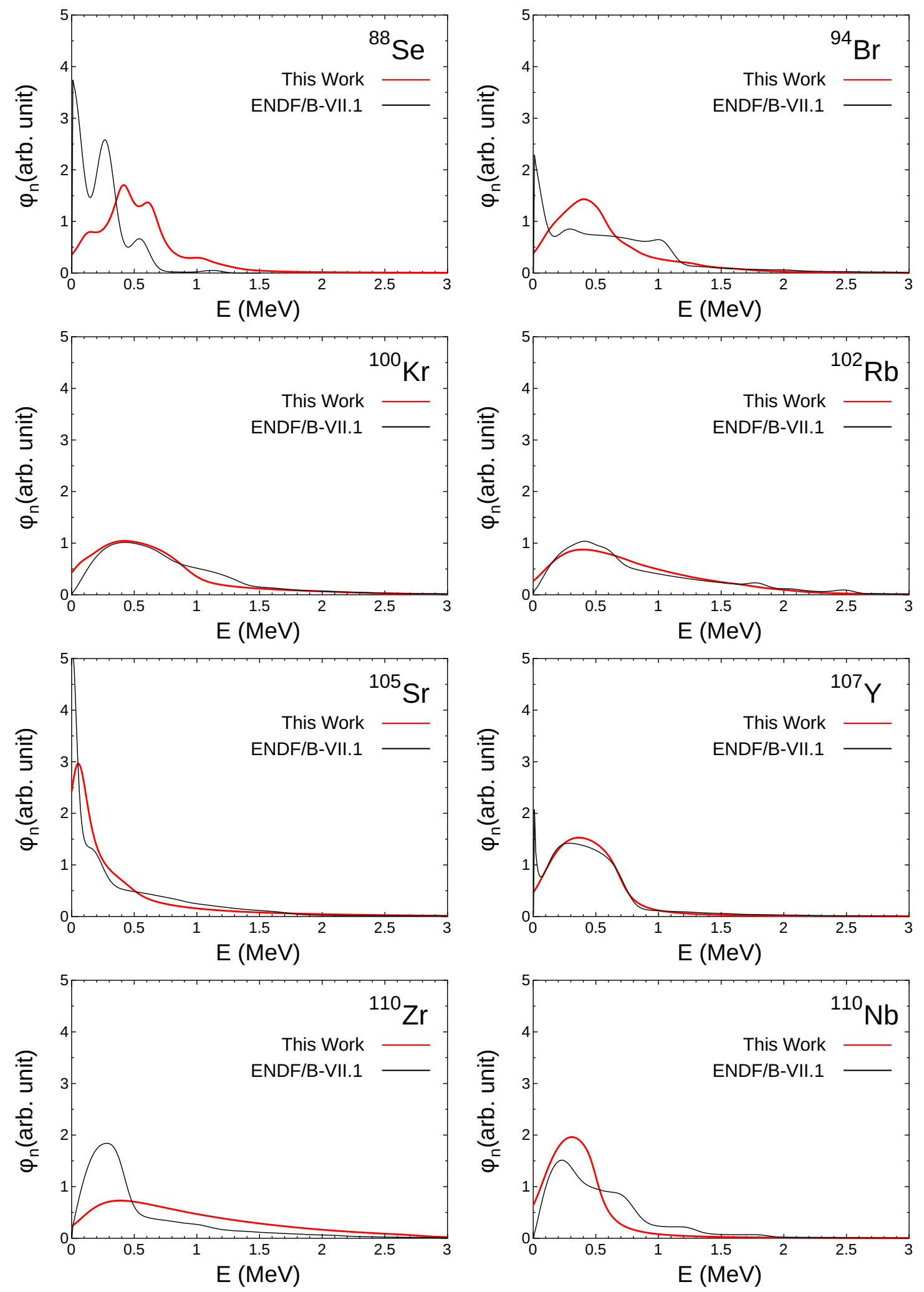

Figure 8. Same as Fig. 7 
will need to include it in future for a more realistic and reliable calculation. In addition, we used the same isoscaler $T=0$ pairing strength $v_{10}$ as the even-even for odd-mass nuclei. It would be more practical to use $v_{10}$ determined for each isotopes individually. To use a mass dependent $v_{10}$ as used in [11] will also improve our result, which is next step of our work.

\section{References}

[1] J. Katakura, JAEA-Data/Code 2011-025 (Mar 2012).

[2] J. Katakura, to be submitted.

[3] M.A. Kellett, O. Bersillon, R.W. Mills, JEFF Report 20, OECD/NEA (2009).

[4] M.B. Chadwick et al., Nucl. Data Sheets 112 (2011) 2887.

[5] J. Katakura, F. Minato, K. Ohgama, to be published in EPJ Web of Conferences, Wonder 2015: Fourth International Workshop on Nuclear Data Evaluation for Reactor Applications, Oct. 2015, Aix-en-Provence, France.

[6] T. Tachibana, M. Yamada, Proc. Inc. Conf. on exotic nuclei and atomic masses, Arles, 1995, eds. M. de Saint Simon and O. Sorlin (Editions Frontueres, Gif-sur-Yvette, 1995) p.763; T. Yoshida and T. Tachibana, J. Nucl. Sci. Technol., 37 (2000) 491.

[7] G. Lorusso et al., Phys. Rev. Lett. 114 (2015) 192501.

[8] F. Minato, O. Iwamoto, JPS Conf. Proc. 6 (2015) 030053.

[9] I.N. Borzov, Nucl. Phys. A777 (2006) 645.

[10] M. Martini, S. Péru, S. Goriely, Phys. Rev. C 89 (2014) 044306.

[11] T. Marketin, L. Huther, G. Martinez-Pinedo, arXiv:1507.07442 (2015).

[12] M.T. Mustonen, J. Engel, arXiv:1510.02136 (2015).

[13] P. Möller, B. Pfeiffer, K.-L. Kratz, Phys. Rev. C 67 (2003) 055802.

[14] T. Kawano, P. Möller, W.B. Wilson, Phys. Rev. C 78 (2008) 054601.

[15] P.-G. Reinhard, D.J. Dean, W. Nazarewicz et al., Phys. Rev. C 60 (1999) 014316.

[16] M. Bender, K. Rutz, P.-G. Reinhard, J.A. Maruhn, Phys. Rev. C 60 (1999) 034304.

[17] O. Iwamoto, J. Nucl. Sci. Tech. 44 (2007) 687.

[18] A.J. Koning, J.P. Delaroche, Nucl. Phys. 713 (2003) 231.

[19] A. Gilbert, A.G.W. Cameron, Can. J. Phys. 43 (1965) 1446.

[20] A. Mengoni, Y. Nakajima, J. Nucl. Sci. Technol. 31 (1994) 151.

[21] P. Ring and P. Schuck, The Nuclear Many Body Problem, Springer, Berlin (1980).

[22] Sara Perez-Martin, L.M. Robledo, Phys. Rev. C 78 (2008) 014304.

[23] National Nuclear Data Center, Brookhaven National Laboratory, NuDat 2.6, http://www.nndc.bnl.gov/nudat2/ 